- Original Article

\title{
The Relationship between Aortic Knob Width and Metabolic Syndrome
}

\author{
Eun-Ji Lee', Jee-Hye Han ${ }^{1, *}$, Kil-Young Kwon', Jung-Hwan Kim', Kun-Hee Han², Si-Yeun Sung', Seo-Rim Hong' \\ 'Department of the Family Medicine, Nowon Eulji Medical Center, Eulji University School of Medicine, Seoul, Korea \\ ${ }^{2}$ Department of the Family Medicine, CHA Bundang Medical Center, CHA University School of Medicine, Seongnam, Korea
}

Background: Both aortic knob width and metabolic syndrome are suggested to be related to atherosclerosis and cardiovascular diseases. However, the association between aortic knob width and metabolic syndrome is unknown. This study aimed to explore this relationship.

Methods: Participants were 3,705 Korean adults aged 18-79 years who visited the health promotion center of a general hospital. Data on chest radiography, physical measurements, medical and social history, and blood tests were collected. We defined metabolic syndrome according to the National Cholesterol Education Program Adult Treatment Panel III criteria. A single reviewer measured aortic knob width on chest radiography.

Results: Aortic knob width was significantly correlated with age; body mass index; waist circumference; systolic and diastolic blood pressures; total cholesterol, triglyceride, high-density lipoprotein cholesterol, low-density lipoprotein cholesterol, fasting glucose, glycated hemoglobin, insulin, and uric acid levels; and homeostatic model assessment of insulin resistance values. Aortic knob width significantly increased as the number of metabolic syndrome components increased. Moreover, metabolic syndrome component values tended to increase across the quartile groups of aortic knob width after adjusting for age, exercise, smoking status, and alcohol use. Through receiver operating characteristic curve analysis, we determined the clinically useful cutoff value for aortic knob width to be $30.47 \mathrm{~mm}$ in premenopausal women.

Conclusion: Aortic knob width was found to be significantly related to metabolic syndrome and its individual components.

Keywords: Metabolic Syndrome; Aortic Knob Width; Thoracic Aorta; Chest Radiography

Received: March 7, 2017, Revised: June 8, 2017, Accepted: July 11, 2017

${ }^{*}$ Corresponding Author: Jee-Hye Han https://orcid.org/0000-0003-4002-3453

Tel: +82-2-970-8515, Fax: +82-2-970-8370, E-mail: hnjh66@hanmail.net 


\section{INTRODUCTION}

Radiography is one of the most inexpensive and easily accessible imaging modalities in the clinical setting. Despite the latest European guidelines on aortic diseases stating that chest radiography is of limited value for diagnosing acute aortic syndrome, ${ }^{1)}$ this modality provides valuable and fundamental information about the heart, the lungs, and bony structures.

The aortic knob width (AKW) is a radiographic configuration formed by a portion of the descending aorta and the foreshortened aortic arch. ${ }^{2)}$ An increase in the transverse diameter of the aortic arch has been associated with atherosclerosis and cardiovascular diseases (CVDs) such as target organ damage, ${ }^{3)}$ hypertension, cardiac dysfunction, aortic calcifications, and coronary artery disease (CAD). ${ }^{4,5}$

Dyslipidemia, diabetes, hypertension, and smoking status are known risk factors for CVDs. ${ }^{6}$ Metabolic syndrome (MetS) is a collection of cardiovascular risk factors that increases cardiovascular mortality and morbidity beyond the sum of risk components. ${ }^{7)}$ As both AKW and MetS are significantly associated with CVDs, we hypothesized that AKW might be related to MetS. Previous studies examined the relationship between AKW and cardio-ankle vascular stiffness index ${ }^{8)}$ or carotid intima-media thickness (CIMT) ${ }^{99}$ However, no studies to date have focused on the relationship between MetS and AKW. This study aimed to explore this relationship.

\section{METHODS}

\section{Subjects}

Study participants were 3,970 Korean adults aged 18-79 years who visited the health promotion center of a general hospital for regular checkups from January to December 2015. The following individuals were excluded from the study: (1) two with structural abnormalities such as mediastinal shift and a history of previous pneumonectomy; 2) 27 with chronic liver diseases ( 3 times the upper normal limits of aspartate aminotransferase/alanine aminotransferase, total bilirubin level >3 mg/dL); (3) eight with acute inflammation (white blood cell count $>13,000 / \mu \mathrm{L}$ ); (4) 101 with any history of cancers; (5) 40 with anemia (hemoglobin level $<9 \mathrm{~g} / \mathrm{dL}$ ); (6) two with chronic renal diseases (estimated glomerular filtration $\operatorname{rate}^{10)}<30 \mathrm{~mL} / \mathrm{min} / 1.73 \mathrm{~m}^{2}$ ); (7) 22 who were pregnant or did not undergo chest radiography; (8) 23 who did not undergo laboratory examination; (9) 46 with abnormal findings on thyroid function tests (thyroid-stimulating hormone level $<0.1$ or $>10 \mu \mathrm{IU} / \mathrm{mL}$ ); and (10) eight with outliers (fasting glucose level $>300$ $\mathrm{mg} / \mathrm{dL}$, insulin level $>300 \mu \mathrm{IU} / \mathrm{mL}$ ). Ultimately, 265 were excluded, leaving a total of 3,705 study participants for this analysis.

This is a retrospective study which does not require the Institutional Review Board approval. And all participants provided written informed consent.

\section{Clinical Examination and Blood Assays}

Lifestyle, social, and medical history data were collected through self- administrated questionnaires. Height and weight were measured using an automatic digital stadiometer. Body mass index (BMI) was defined as body weight divided by height squared $\left(\mathrm{kg} / \mathrm{m}^{2}\right)$. Blood pressure (BP) was measured using an automated sphygmomanometer after the participants were comfortably seated for at least 10 minutes. Blood samples were drawn from the antecubital vein in the morning after fasting for more than 8 hours. Laboratory tests for total cholesterol, triglyceride, high-density lipoprotein (HDL) cholesterol, low-density lipoprotein (LDL) cholesterol, fasting glucose, insulin, uric acid, creatinine, and glycated hemoglobin (HbAlc) levels were performed. Homeostatic model assessment of insulin resistance (HOMA-IR) was calculated using standard methods. ${ }^{11)}$

MetS, in accordance with the National Cholesterol Education Program Adult Treatment Panel III criteria, ${ }^{12-14)}$ was defined as the presence of three or more of the following: elevated triglyceride level $(\geq 150$ $\mathrm{mg} / \mathrm{dL}$ or use of triglyceride-lowering medications), low HDL cholesterol level (men $<40 \mathrm{mg} / \mathrm{dL}$, women $<50 \mathrm{mg} / \mathrm{dL}$, or use of HDL cholesterol-raising medications), elevated systolic BP (SBP) or diastolic BP (DBP) ( $\geq 130 / 85 \mathrm{~mm}$ Hg or use of antihypertensive medications), elevated fasting glucose level $(\geq 100 \mathrm{mg} / \mathrm{dL}$ or use of antidiabetic medications), and increased waist circumference ( $\geq 90 \mathrm{~cm}$ in men, $\geq 85 \mathrm{~cm}$ in women). ${ }^{13)}$

\section{Measurement of Aortic Knob Width}

On posteroanterior chest radiography, the widest point of the aortic knob was measured along the straight imaginary line from the lateral edge of the trachea to the left lateral wall of the aortic arch. ${ }^{2,4,8,9,15,16)}$ To minimize interpersonal differences, a single reviewer blinded to the participants' demographic data measured all AKW on chest radiography.

\section{Statistical Analysis}

All continuous variables except for triglyceride and insulin levels were analyzed using independent t-tests, whereas categorical variables were compared using chi-square test. Because triglyceride and insulin levels did not pass the normality tests, they were subjected to the Mann-Whitney U-test. Pearson correlation analysis was used to determine the association between metabolic parameters and AKW. For the same reason, triglyceride and insulin levels were determined using nonparametric Spearman correlation coefficients. To evaluate the AKW trends according to the number of MetS components and each MetS component according to AKW quartile, the P for trend was determined using a contrast test for the analysis of covariance (ANCOVA) after adjusting for age, alcohol use, smoking status, and exercise. Receiver operating characteristic (ROC) curves were used to assess the sensitivity and specificity of AKW to identify MetS. All statistical analyses were performed using PASW SPSS for Windows ver. 18.0 (SPSS Inc., Chicago, IL, USA). 


\section{RESULTS}

The study included 2,002 men (54.04\%) and 1,703 women (45.96\%) aged 18-79 years. Mean AKW was $32.29 \pm 5.30 \mathrm{~mm}$ overall, $34.21 \pm 4.78$ $\mathrm{mm}$ in men, and $30.03 \pm 4.98 \mathrm{~mm}$ in women. The mean age was $45.45 \pm 11.56$ years, and the mean BMI was $23.88 \pm 3.35 \mathrm{~kg} / \mathrm{m}^{2}$ (Table 1 ).

AKW showed significant correlations with age; BMI; waist circumference; SBP; DBP; total cholesterol, triglyceride, HDL cholesterol, LDL cholesterol, fasting glucose, insulin, $\mathrm{HbAlc}$, and uric acid levels; and HOMA-IR values (Table 2). All variables showed significant correlations with AKW, except for total cholesterol and uric acid levels in men and insulin level in women.

Analysis of variance showed a significant increase in AKW in accordance with the number of MetS components (Table 3). Moreover, AN-
COVA after adjusting for age, exercise, smoking status, and alcohol use showed a significant increase in each MetS component according to AKW quartile (Table 4).

In the ROC curve analysis of the ability of AKW to predict MetS, we divided men into three groups based on age and women into two groups, considering 50 years as the average menopause age. The cutoff values were $33.49 \mathrm{~mm}$ overall (sensitivity, $68 \%$; specificity, $67 \%$; area under the curve [AUC], 0.74; 95\% confidence interval [CI], 0.72-0.76; $\mathrm{P}<0.001$ ) (Figure 1A), $32.05 \mathrm{~mm}$ in men aged 20-39 years (sensitivity, 52\%; specificity, 69\%; AUC, 0.61; 95\% CI, 0.53-0.68; $\mathrm{P}<0.001$ ) (Figure 1B), $34.39 \mathrm{~mm}$ in men aged $40-59$ years (sensitivity, $64 \%$; specificity, 57\%; AUC, 0.64; 95\% CI, 0.60-0.67; $\mathrm{P}<0.001$ ) (Figure 1C), $36.98 \mathrm{~mm}$ in men aged 60-79 years (sensitivity, $80 \%$; specificity, $40 \%$; AUC, 0.58 ; 95\% CI, 0.50-0.66; $\mathrm{P}<0.001$ ) (Figure 1D), $30.47 \mathrm{~mm}$ in women aged $20-$

Table 1. Characteristics of study participants

\begin{tabular}{|c|c|c|c|c|}
\hline Characteristic & Total $(n=3,705)$ & Men $(n=2,002)$ & Women $(n=1,703)$ & P-value \\
\hline Aortic knob width (mm) & $32.29 \pm 5.30$ & $34.21 \pm 4.78$ & $30.03 \pm 4.98$ & $<0.001$ \\
\hline Age (y) & $45.45 \pm 11.56$ & $46.82 \pm 10.68$ & $43.84 \pm 12.32$ & $<0.001$ \\
\hline Body mass index $\left(\mathrm{kg} / \mathrm{m}^{2}\right)$ & $23.88 \pm 3.35$ & $24.96 \pm 2.97$ & $22.61 \pm 3.31$ & $<0.001$ \\
\hline Waist circumference (cm) & $80.52 \pm 10.30$ & $85.80 \pm 7.97$ & $74.30 \pm 9.21$ & $<0.001$ \\
\hline Systolic blood pressure (mm Hg) & $118.15 \pm 13.43$ & $121.38 \pm 12.71$ & $114.35 \pm 13.27$ & $<0.001$ \\
\hline Diastolic blood pressure (mm Hg) & $71.50 \pm 9.86$ & $73.65 \pm 9.49$ & $68.97 \pm 9.68$ & $<0.001$ \\
\hline Total cholesterol level (mg/dL) & $201.79 \pm 36.87$ & $205.50 \pm 38.52$ & $197.43 \pm 34.33$ & $<0.001$ \\
\hline Triglyceride level (mg/dL) & $120.54 \pm 106.84$ & $148.36 \pm 128.88$ & $87.85 \pm 57.75$ & $<0.001$ \\
\hline High-density lipoprotein cholesterol level (mg/dL) & $56.46 \pm 13.54$ & $51.16 \pm 11.32$ & $62.68 \pm 13.29$ & $<0.001$ \\
\hline Low-density lipoprotein cholesterol level (mg/dL) & $121.71 \pm 33.03$ & $127.16 \pm 33.49$ & $115.30 \pm 31.30$ & $<0.001$ \\
\hline Fasting glucose level (mg/dL) & $91.50 \pm 20.55$ & $94.50 \pm 23.25$ & $87.98 \pm 16.15$ & $<0.001$ \\
\hline Hemoglobin A1c level (\%) & $5.64 \pm 0.72$ & $5.72 \pm 0.80$ & $1.09 \pm 2.14$ & $<0.001$ \\
\hline Insulin level ( $\mu \mathrm{U} \mathrm{U} / \mathrm{mL})$ & $4.92 \pm 5.72$ & $5.04 \pm 4.03$ & $4.77 \pm 7.25$ & $<0.001$ \\
\hline Homeostatic model assessment of insulin resistance & $1.16 \pm 1.68$ & $1.23 \pm 1.16$ & $1.09 \pm 2.14$ & 0.020 \\
\hline Uric acid level (mg/dL) & $5.21 \pm 1.37$ & $5.94 \pm 1.25$ & $4.33 \pm 0.91$ & $<0.001$ \\
\hline Current smoker & $790(21.3)$ & $737(36.8)$ & $53(3.1)$ & $<0.001$ \\
\hline
\end{tabular}

Values are presented as mean \pm standard deviation or number (\%). All data except for triglyceride and insulin levels were analyzed by independent t-tests. Triglyceride and insulin levels were analyzed by Mann-Whitney U-test. P-value refers to comparison between men and women.

Table 2. Correlations between aortic knob width and variables

\begin{tabular}{|c|c|c|c|c|c|c|}
\hline \multirow{2}{*}{ Variable } & \multicolumn{2}{|c|}{ Total } & \multicolumn{2}{|c|}{ Men } & \multicolumn{2}{|c|}{ Women } \\
\hline & $\mathrm{R}$ & P-value & $\mathrm{R}$ & P-value & $\mathrm{R}$ & P-value \\
\hline Age (y) & 0.616 & $<0.001$ & 0.547 & $<0.001$ & 0.694 & $<0.001$ \\
\hline Body mass index $\left(\mathrm{kg} / \mathrm{m}^{2}\right)$ & 0.408 & $<0.001$ & 0.256 & $<0.001$ & 0.374 & $<0.001$ \\
\hline Waist circumference (cm) & 0.490 & $<0.001$ & 0.294 & $<0.001$ & 0.416 & $<0.001$ \\
\hline Systolic blood pressure (mm Hg) & 0.352 & $<0.001$ & 0.202 & $<0.001$ & 0.367 & $<0.001$ \\
\hline Diastolic blood pressure (mm Hg) & 0.330 & $<0.001$ & 0.216 & $<0.001$ & 0.319 & $<0.001$ \\
\hline Total cholesterol level (mg/dL) & 0.100 & $<0.001$ & -0.022 & 0.317 & 0.170 & $<0.001$ \\
\hline Triglyceride level (mg/dL) & 0.319 & $<0.001$ & 0.113 & $<0.001$ & 0.237 & $<0.001$ \\
\hline High-density lipoprotein cholesterol level (mg/dL) & -0.279 & $<0.001$ & -0.080 & $<0.001$ & -0.187 & 0.001 \\
\hline Low-density lipoprotein cholesterol level (mg/dL) & 0.118 & $<0.001$ & -0.053 & 0.017 & 0.182 & $<0.001$ \\
\hline Fasting glucose level (mg/dL) & 0.244 & $<0.001$ & 0.168 & $<0.001$ & 0.263 & $<0.001$ \\
\hline Insulin level $(\mu \mid \mathrm{U} / \mathrm{mL})$ & 0.104 & $<0.001$ & 0.086 & $<0.001$ & 0.032 & 0.215 \\
\hline Homeostatic model assessment of insulin resistance & 0.094 & $<0.001$ & 0.115 & $<0.001$ & 0.073 & 0.005 \\
\hline Hemoglobin A1c (\%) & 0.250 & $<0.001$ & 0.149 & $<0.001$ & 0.332 & $<0.001$ \\
\hline Uric acid level (mg/dL) & 0.247 & $<0.001$ & 0.000 & 0.983 & 0.054 & 0.029 \\
\hline
\end{tabular}

All values except for triglyceride and insulin levels are presented as correlation coefficient by Pearson correlation analysis. Triglyceride and insulin levels are presented as nonparametric Spearman correlation coefficients. 
Table 3. Trends of aortic knob width according to the number of MetS components

\begin{tabular}{|c|c|c|c|c|c|c|}
\hline \multirow{2}{*}{ Variable } & \multicolumn{4}{|c|}{ No. of MetS components } & \multirow{2}{*}{ P-value } & \multirow{2}{*}{ P for trenc } \\
\hline & 0 & 1 & 2 & $\geq 3$ & & \\
\hline Total (mm) & $29.73 \pm 4.51(1,577)$ & $33.05 \pm 4.69(864)$ & $34.06 \pm 5.01(671)$ & $35.98 \pm 5.06(593)$ & $<0.001$ & $<0.001$ \\
\hline Men (mm) & $32.25 \pm 4.24(551)$ & $34.16 \pm 4.39(529)$ & $34.59 \pm 4.69(467)$ & $36.26 \pm 4.98$ (455) & $<0.001$ & $<0.001$ \\
\hline Women (mm) & $28.39 \pm 4.06(1,026)$ & $31.30 \pm 4.61(335)$ & $32.84 \pm 5.49(204)$ & $35.04 \pm 5.22(138)$ & $<0.001$ & $<0.001$ \\
\hline
\end{tabular}

Values are presented as mean \pm standard deviation (number). Analyzed by ANCOVA after adjusting for age, alcohol use, smoking status, and exercise. $\mathrm{P}$ for trend was determined using a contrast test for ANCOVA.

MetS, metabolic syndrome; ANCOVA, analysis of covariance.

Table 4. Trends of each metabolic syndrome component according to AKW quartile

\begin{tabular}{|c|c|c|c|c|c|c|}
\hline Variable & 1st AKW quartile & 2nd AKW quartile & 3rd AKW quartile & 4th AKW quartile & P-value & P for trend \\
\hline Total & $14.16-28.49 \mathrm{~mm}$ & $28.50-32.00 \mathrm{~mm}$ & $32.01-35.60 \mathrm{~mm}$ & $35.61-75.00 \mathrm{~mm}$ & & \\
\hline Waist circumference (cm) & $74.38 \pm 0.35$ & $79.51 \pm 0.29$ & $82.49 \pm 0.30$ & $86.04 \pm 0.33$ & $<0.001$ & $<0.001$ \\
\hline $\mathrm{SBP}(\mathrm{mm} \mathrm{Hg})$ & $112.01 \pm 0.49$ & $116.23 \pm 0.42$ & $119.88 \pm 0.43$ & $124.38 \pm 0.47$ & $<0.001$ & $<0.001$ \\
\hline $\mathrm{DBP}(\mathrm{mm} \mathrm{Hg})$ & $66.49 \pm 0.36$ & $69.98 \pm 0.31$ & $73.44 \pm 0.31$ & $76.04 \pm 0.35$ & $<0.001$ & $<0.001$ \\
\hline Triglyceride level (mg/dL) & $95.16 \pm 4.08$ & $120.45 \pm 3.48$ & $130.04 \pm 3.52$ & $139.22 \pm 3.88$ & $<0.001$ & $<0.001$ \\
\hline HDL cholesterol level (mg/dL) & $62.22 \pm 0.50$ & $56.32 \pm 0.43$ & $54.10 \pm 0.43$ & $53.07 \pm 0.48$ & $<0.001$ & $<0.001$ \\
\hline Fasting glucose level (mg/dL) & $88.96 \pm 0.79$ & $90.06 \pm 0.68$ & $92.10 \pm 0.69$ & $95.29 \pm 0.76$ & $<0.001$ & $<0.001$ \\
\hline Men & $21.40-30.89 \mathrm{~mm}$ & $30.90-33.79 \mathrm{~mm}$ & $33.80-37.19 \mathrm{~mm}$ & $37.20-75.00 \mathrm{~mm}$ & & \\
\hline Waist circumference $(\mathrm{cm})$ & $82.69 \pm 0.38$ & $84.81 \pm 0.35$ & $86.30 \pm 0.35$ & $89.55 \pm 0.38$ & $<0.001$ & $<0.001$ \\
\hline $\mathrm{SBP}(\mathrm{mm} \mathrm{Hg})$ & $117.94 \pm 0.61$ & $119.63 \pm 0.56$ & $122.62 \pm 0.56$ & $125.28 \pm 0.60$ & $<0.001$ & $<0.001$ \\
\hline $\mathrm{DBP}(\mathrm{mm} \mathrm{Hg})$ & $70.01 \pm 0.45$ & $72.44 \pm 0.41$ & $75.49 \pm 0.42$ & $76.52 \pm 0.44$ & $<0.001$ & $<0.001$ \\
\hline Triglyceride level (mg/dL) & $131.65 \pm 6.33$ & $139.98 \pm 5.83$ & $165.16 \pm 5.86$ & $158.89 \pm 6.25$ & 0.001 & $<0.001$ \\
\hline HDL cholesterol level (mg/dL) & $52.95 \pm 0.55$ & $51.39 \pm 0.50$ & $50.63 \pm 0.51$ & $49.72 \pm 0.54$ & 0.001 & $<0.001$ \\
\hline Fasting glucose level (mg/dL) & $92.50 \pm 1.14$ & $92.80 \pm 1.05$ & $95.99 \pm 1.05$ & $97.15 \pm 1.12$ & 0.011 & 0.002 \\
\hline Women & $14.16-26.49 \mathrm{~mm}$ & $26.50-29.59 \mathrm{~mm}$ & $29.60-33.02 \mathrm{~mm}$ & $33.03-64.14 \mathrm{~mm}$ & & \\
\hline Waist circumference (cm) & $71.18 \pm 0.49$ & $72.89 \pm 0.43$ & $74.65 \pm 0.43$ & $78.24 \pm 0.49$ & $<0.001$ & $<0.001$ \\
\hline $\mathrm{SBP}(\mathrm{mm} \mathrm{Hg})$ & $110.70 \pm 0.72$ & $111.55 \pm 0.63$ & $113.96 \pm 0.63$ & $120.58 \pm 0.72$ & $<0.001$ & $<0.001$ \\
\hline $\mathrm{DBP}(\mathrm{mm} \mathrm{Hg})$ & $65.23 \pm 0.53$ & $67.24 \pm 0.47$ & $69.70 \pm 0.47$ & $73.54 \pm 0.53$ & $<0.001$ & $<0.001$ \\
\hline Triglyceride level (mg/dL) & $82.50 \pm 3.15$ & $81.08 \pm 2.78$ & $92.84 \pm 2.76$ & $93.24 \pm 3.14$ & 0.008 & $<0.001$ \\
\hline HDL cholesterol level (mg/dL) & $65.39 \pm 0.75$ & $63.91 \pm 0.66$ & $61.30 \pm 0.66$ & $60.70 \pm 0.75$ & $<0.001$ & $<0.001$ \\
\hline Fasting glucose level (mg/dL) & $85.40 \pm 0.90$ & $86.02 \pm 0.79$ & $88.23 \pm 0.79$ & $92.13 \pm 0.89$ & $<0.001$ & $<0.001$ \\
\hline
\end{tabular}

Values are presented as range or mean \pm standard deviation, unless otherwise stated. Analyzed by ANCOVA after adjusting for age, alcohol use, smoking status, and exercise. $P$ for trend was determined using a contrast test for ANCOVA.

AKW, aortic knob width; SBP, systolic blood pressure; DBP, diastolic blood pressure; HDL, high-density lipoprotein; ANCOVA, analysis of covariance.

49 years (sensitivity, 0.76\%; specificity, 0.75\%; AUC, 0.79; $95 \%$ CI, $0.73-$ 0.85 ; $\mathrm{P}<0.001$ ) (Figure $1 \mathrm{E}$ ), and $36.14 \mathrm{~mm}$ in women aged $50-69$ years (sensitivity, 0.50\%; specificity, 0.76\%; AUC, 0.66; 95\% CI, 0.60-0.73; $\mathrm{P}<0.001$ ) (Figure $1 \mathrm{~F}$ ). Considering the area under the ROC curve (i.e., AUC) values of $0.7-0.8$ as fair and clinically useful, ${ }^{17,18)}$ the discriminatory power of AKW was moderate in premenopausal women.

\section{DISCUSSION}

This study showed that AKW was significantly correlated with multiple factors, including older age, higher BMI, abdominal obesity, hypertension, dyslipidemia, and insulin resistance. AKW showed an increasing trend based on the number of MetS components, and each MetS component showed increasing trends according to AKW quartile. An exception for this was the triglyceride level in several AKW quartile subgroups in men and women, but the overall trends were statistically significant. In addition, we confirmed moderate clinical usefulness for distinguishing MetS in premenopausal women with a cutoff value of $30.47 \mathrm{~mm}$ for AKW.

The normal human adult aortic wall comprises three layers: intima, media, and adventitia. ${ }^{19)}$ Atherosclerosis is characterized by intimal lesions called atheromata, or atheromatous or fibrofatty plaques, which protrude into the arterial lumen and weaken the underlying media often associated with calcification. With aging, presence of risk factors, and genetic predisposition, ${ }^{20)}$ this condition progresses to complicated lesions with surface defects, hemorrhage, and/or thrombosis. ${ }^{21)}$ The aortic knob can be enlarged because of increased pressure flow in the aorta or changes in the elasticity of its wall. ${ }^{20)}$

A few similar studies have been published. One Japanese study involving hypertensive patients with known or suspected CAD suggested that AKW is an independent predictor of central SBP. ${ }^{15)} \mathrm{A}$ Turkish study that compared AKW with cardio-ankle vascular stiffness index in the diagnosis of subclinical atherosclerosis suggested a cutoff value of $41 \mathrm{~mm}$ for AKW to detect subclinical atherosclerosis. ${ }^{8)}$ Another 
Turkish study that compared AKW with CIMT in hypertensive patients showed a strong relationship between them. AKW, age, and SBP were also reported as independent predictors of CIMT.9) A French study that focused on the relationship between MetS and age-related progres-
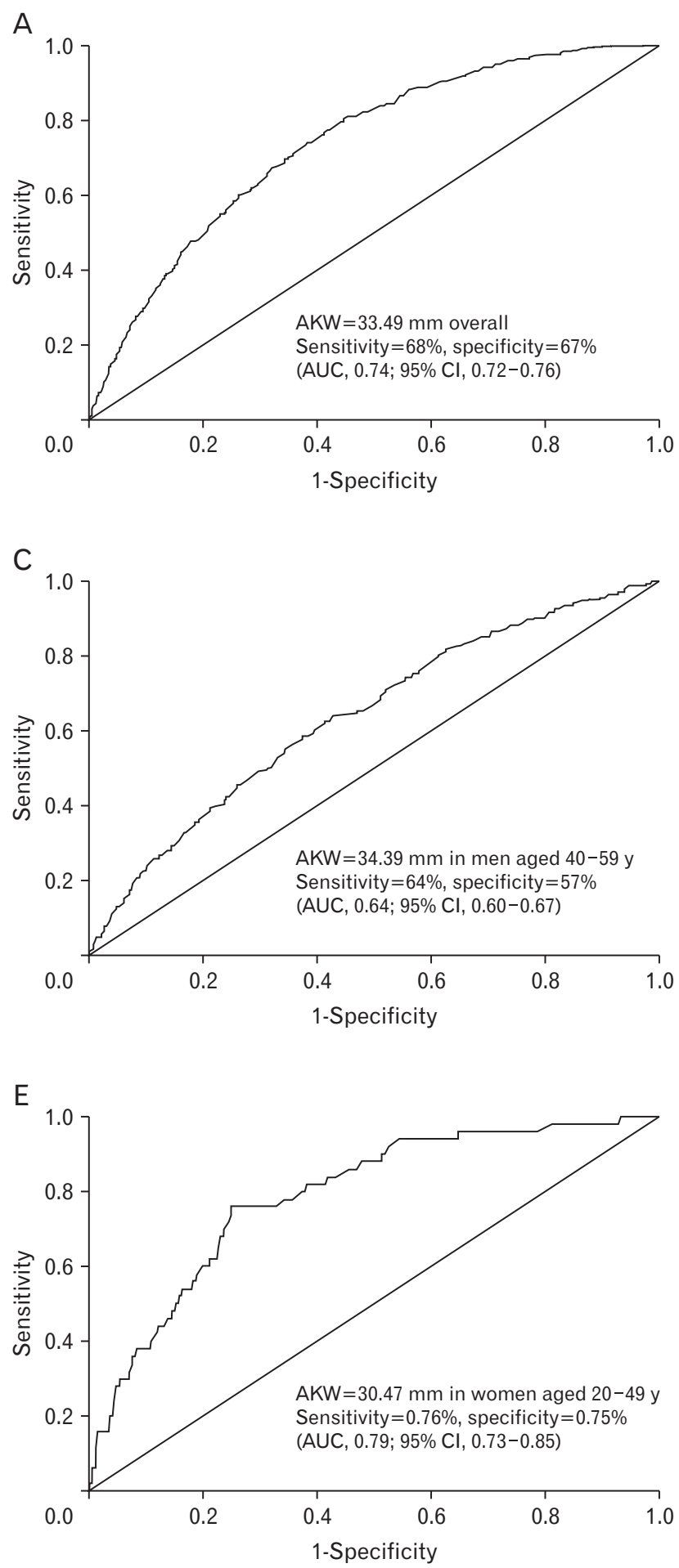

sion of aortic stiffness measured using aortic pulse wave velocity showed a positive result. ${ }^{22)}$

A number of factors, including age, sex, body size, measurement method, and imaging methods used, affect "normal" aortic diame-
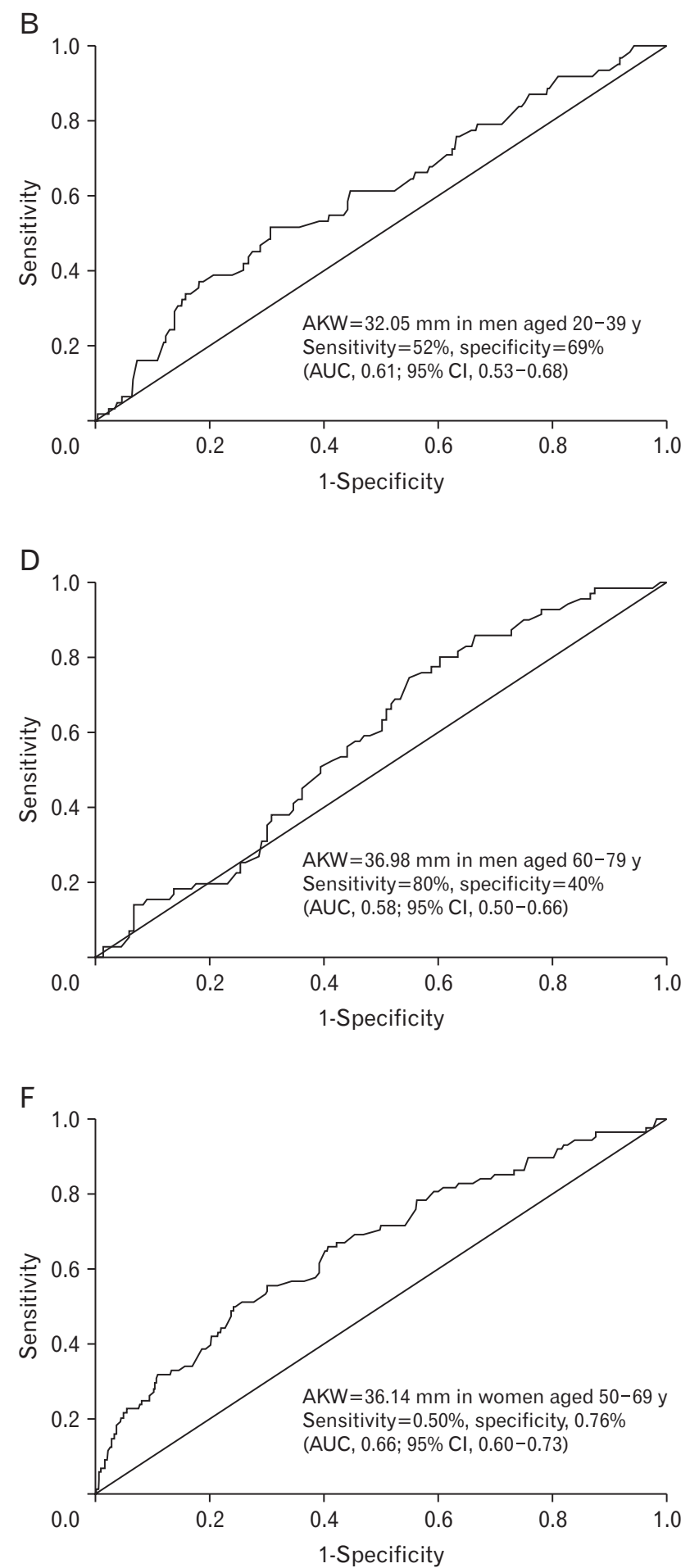

Figure 1. ROC curves in specified participants. (A) ROC curve in all participants. (B) ROC curve in men aged from 20 to 39. (C) ROC curve in men aged from 40 to 59 . (D) ROC curve in men aged from 60 to 79. (E) ROC curve in women aged from 20 to 49. (F) ROC curve in women aged from 50 to 79 . AKW, aortic knob width; ROC, receiver operating characteristic; AUC, area under the curve; $\mathrm{Cl}$, confidence interval. 
ter. ${ }^{21)}$ In healthy adults, aortic diameters are usually $<40 \mathrm{~mm}$ and are larger in men, and they increase with age $(0.9 \mathrm{~mm}$ in men and $0.7 \mathrm{~mm}$ in women for each decade of life), body size (height, weight, body surface area), ${ }^{1,21)}$ and BP. ${ }^{1)}$ One previous study with a small sample size reported that the mean AKW was $3.16 \pm 0.60 \mathrm{~cm}$ in men and $2.75 \pm 0.35 \mathrm{~cm}$ in women, with AKW differently defined as the minimum distance of the left edge of the aortic knob from the midline. ${ }^{23)}$ Another cross-sectional study on an Indian population indicated that the mean AKW was $3.10 \pm 3.34 \mathrm{~cm}$ in men and $3.08 \pm 3.90 \mathrm{~cm}$ in women and that increased with age. ${ }^{20)}$ Another Turkish study involving patients on hemodialysis reported that AKW was independently related to male sex, age, and $\mathrm{SBP}^{24)}$ In our study, AKW was greater in men than in women and increased with age, BMI, waist circumference, SBP, and DBP, which was similar to the results of previous studies.

After adjusting for age, alcohol use, smoking status, and exercise, significant increases in each MetS component according to AKW quartile in both men and women were observed. Moreover, AKW increased as the number of MetS components increased. These results support that MetS and each of its individual components were related to AKW.

The discriminatory ability of AKW to identify MetS was fair in premenopausal women and overall in this study. However, it seemed less useful in men and postmenopausal women (AUC <0.70). This suggested that AKW is a surrogate marker for MetS in premenopausal women.

Structural changes in the aorta seem irreversible, especially when calcifications develop, and patients with MetS were emphasized as a high-risk group for the development of CVDs. ${ }^{12)}$ Early identification of MetS is important for the preparation of decisive strategies for managing probable future major adverse cardiovascular events. ${ }^{25)}$ In particular, in clinical settings in which no laboratory tests but chest radiography are available, AKW could be helpful for predicting MetS.

The latest guidelines on aortic diseases recommend the use of computed tomography and/or magnetic resonance imaging for assessing external aortic diameter and echocardiography for assessing internal aortic diameter. ${ }^{1)}$ Moreover, minimizing radiation exposure was heavily emphasized. ${ }^{21)}$ From this point of view, chest radiography is the most cost-effective radiologic test with minimal concern for radiationinduced malignancy in the clinical setting. Therefore, for primary physicians, measuring AKW on routine chest radiography is cost-effective and safe enough for detecting MetS when it is clinically doubtful.

The limitations of this study must be considered. First, its cross-sectional design makes it difficult to demonstrate causal relationships. Second, selection bias was unavoidable owing to the use of a single center. Third, the participants might have paid closer attention to their health than the general population because they voluntarily attended a health checkup.

Nevertheless, this study also has important strengths. First, it was the first study designed to show the relationship between AKW and MetS. Second, the study population was comparatively large and included a high percentage of healthy adults without underlying disease.
Most previous studies on AKW were confined to hypertensive individuals or those at risk for CVDs. ${ }^{3,4,8,9,24)}$

In conclusion, this study showed a significant relationship between AKW and MetS. Chest radiography is cost-effective and readily accessible with minimal concern related to excessive radiation exposure. AKW is easily measured by primary physicians. Therefore, they can pay more attention to patients with a greater AKW than the cutoff values in this study on routine chest radiography, particularly in premenopausal women.

\section{CONFLICT OF INTEREST}

No potential conflict of interest relevant to this article was reported.

\section{REFERENCES}

1. Erbel R, Aboyans V, Boileau C, Bossone E, Bartolomeo RD, Eggebrecht $\mathrm{H}$, et al. 2014 ESC guidelines on the diagnosis and treatment of aortic diseases: document covering acute and chronic aortic diseases of the thoracic and abdominal aorta of the adult. The Task Force for the Diagnosis and Treatment of Aortic Diseases of the European Society of Cardiology (ESC). Eur Heart J 2014;35:2873-926.

2. Erdem K, Ozturk S, Ayhan S, Bugra O, Bozoglan O, Tekelioglu UY, et al. Predictive value of aortic knob width for postoperative atrial fibrillation in coronary artery bypass surgery. Anadolu Kardiyol Derg 2014; 14:68-72.

3. Rayner BL, Goodman H, Opie LH. The chest radiograph: a useful investigation in the evaluation of hypertensive patients. Am J Hypertens 2004;17:507-10.

4. Yun KH, Jeong MH, Oh SK, Park EM, Kim YK, Rhee SJ, et al. Clinical significance of aortic knob width and calcification in unstable angina. Circ J 2006;70:1280-3.

5. Anyanwu GE, Anibeze CI, Akpuaka FC. Transverse aortic arch diameters and relationship with heart size of Nigerians within the South East. Biomed Res 2007;18:115-8.

6. US Preventive Services Task Force, Bibbins-Domingo K, Grossman DC, Curry SJ, Davidson KW, Epling JW Jr, et al. Statin use for the primary prevention of cardiovascular disease in adults: US Preventive Services Task Force recommendation statement. JAMA 2016;316: 1997-2007.

7. Isomaa B, Almgren P, Tuomi T, Forsen B, Lahti K, Nissen M, et al. Cardiovascular morbidity and mortality associated with the metabolic syndrome. Diabetes Care 2001;24:683-9.

8. Korkmaz L, Erkan H, Korkmaz AA, Acar Z, Agac MT, Bektas H, et al. Relationship of aortic knob width with cardio-ankle vascular stiffness index and its value in diagnosis of subclinical atherosclerosis in hypertensive patients: a study on diagnostic accuracy. Anadolu Kardiyol Derg 2012;12:102-6.

9. Erkan H, Korkmaz L, Agaç MT, Acar Z, Kiris A, Erkan M, et al. Relation between carotid intima-media thickness and aortic knob width in patients with essential hypertension. Blood Press Monit 2011;16:282-4.

10. Levey AS, Stevens LA, Schmid CH, Zhang YL, Castro AF 3rd, Feldman $\mathrm{HI}$, et al. A new equation to estimate glomerular filtration rate. Ann In- 
tern Med 2009;150:604-12.

11. Matthews DR, Hosker JP, Rudenski AS, Naylor BA, Treacher DF, Turner RC. Homeostasis model assessment: insulin resistance and beta-cell function from fasting plasma glucose and insulin concentrations in man. Diabetologia 1985;28:412-9.

12. National Cholesterol Education Program (NCEP) Expert Panel on Detection, Evaluation, and Treatment of High Blood Cholesterol in Adults (Adult Treatment Panel III). Third report of the National Cholesterol Education Program (NCEP) Expert Panel on Detection, Evaluation, and Treatment of High Blood Cholesterol in Adults (Adult Treatment Panel III) final report. Circulation 2002;106:3143-421.

13. Lee SY, Park HS, Kim DJ, Han JH, Kim SM, Cho GJ, et al. Appropriate waist circumference cutoff points for central obesity in Korean adults. Diabetes Res Clin Pract 2007;75:72-80.

14. Grundy SM, Cleeman JI, Daniels SR, Donato KA, Eckel RH, Franklin BA, et al. Diagnosis and management of the metabolic syndrome: an American Heart Association/National Heart, Lung, and Blood Institute Scientific Statement. Circulation 2005;112:2735-52.

15. Higaki T, Kurisu S, Watanabe N, Ikenaga H, Shimonaga T, Iwasaki T, et al. Usefulness of aortic knob width on chest radiography to predict central hemodynamics in patients with known or suspected coronary artery disease. Clin Exp Hypertens 2015;37:440-4.

16. Torun E, Kacmaz F. Clinical implications of chest X-ray parameters in evaluating patients with cardiac dyspnea. Eurasian J Med 2008;40:1336.

17. Hanley JA. Receiver operating characteristic (ROC) curves. In: Armitage P, Colton T, editors. Encyclopedia of biostatistics. 2nd ed. Chichester: John Wiley \& Sons; 2005.

18. Hanley JA, McNeil BJ. The meaning and use of the area under a receiver operating characteristic (ROC) curve. Radiology 1982;143:29-36.

19. Milewicz DM, Guo DC, Tran-Fadulu V, Lafont AL, Papke CL, Inamoto
S, et al. Genetic basis of thoracic aortic aneurysms and dissections: focus on smooth muscle cell contractile dysfunction. Annu Rev Genomics Hum Genet 2008;9:283-302.

20. Ray A, Mandal D, Kundu P, Manna S, Mandal S. Aortic knob diameter in chest X-ray and its relation with age, heart diameter and transverse diameter of thorax in a population of Bankura District of West Bengal, India: a cross sectional study. J Evol Med Dent Sci 2014;3:8595-600.

21. Hiratzka LF, Bakris GL, Beckman JA, Bersin RM, Carr VF, Casey DE Jr, et al. $2010 \mathrm{ACCF} / \mathrm{AHA} / \mathrm{AATS} / \mathrm{ACR} / \mathrm{ASA} / \mathrm{SCA} / \mathrm{SCAI} / \mathrm{SIR} / \mathrm{STS} / \mathrm{SVM}$ guidelines for the diagnosis and management of patients with thoracic aortic disease: a report of the American College of Cardiology Foundation/American Heart Association Task Force on Practice Guidelines, American Association for Thoracic Surgery, American College of Radiology, American Stroke Association, Society of Cardiovascular Anesthesiologists, Society for Cardiovascular Angiography and Interventions, Society of Interventional Radiology, Society of Thoracic Surgeons, and Society for Vascular Medicine. J Am Coll Cardiol 2010;55: e27-e129.

22. Safar ME, Thomas F, Blacher J, Nzietchueng R, Bureau JM, Pannier B, et al. Metabolic syndrome and age-related progression of aortic stiffness. J Am Coll Cardiol 2006;47:72-5.

23. Shankar N, Veeramani R, Ravindranath R, Philip B. Anatomical variations of the aortic knob in chest radiographs. Eur J Anat 2010;14:25-30.

24. Afsar B, Saglam M, Yuceturk C, Agca E. The relationship between aortic knob width and various demographic, clinical, and laboratory parameters in stable hemodialysis patients. Saudi J Kidney Dis Transpl 2014;25:1178-85.

25. Georgiopoulos G, Tsioufis C, Tsiachris D, Dimitriadis K, Kasiakogias A, Lagiou F, et al. Metabolic syndrome, independent of its components, affects adversely cardiovascular morbidity in essential hypertensives. Atherosclerosis 2016;244:66-72. 